\title{
MELANOMA OF THE CHOROID EXAMINED WITH AN ACOUSTIC BIOMICROSCOPE*
}

\author{
BY \\ ARVO OKSALA \\ From the Ophthalmic Department of the Central-Finland Regional Hospital, Jyväskylä, Finland
}

ECHOGRAMS have only recently been used in diagnosing intra-ocular tumours (Mundt and Hughes, 1956; Oksala and Lehtinen, 1957; Oksala, 1958, 1959a; Baum and Greenwood, 1958, 1960). According to the observations of Oksala, ultrasonic examination is a valuable addition to the methods of diagnosing this condition.

Since the ultrasound easily penetrates both the transparent and the opaque parts of the eye, we can carry on our examination by ultrasound from the point at which the ray of light has stopped. On the basis of echograms we can evaluate the acoustic properties of the subretinal space compared with those of the vitreous and of the posterior wall of the eye. We can decide with a high degree of certainty whether subretinal fluid or an acoustically much more heterogeneous tumour tissue is present. The intra-ocular tumour is clearly visualized on the echogram only after it has penetrated into the vitreous for at least $2 \mathrm{~mm}$. (Oksala, 1959b).

At the end of 1959 we adopted at our Ophthalmic Department the use of the so-called acoustic biomicroscopic examination (Oksala and Lehtinen, 1960), in which the echogram registered by an ultrasound equipment is enlarged by means of a high-frequency oscilloscope. Since I have not previously published any observations on the examination of intra-ocular tumours by this method, the new research equipment and a typical case are discussed below.

\section{Equipment and Method of Use}

Our research equipment comprises the following pieces of apparatus:

(1) Ultrasonic equipment with crystal†;

(2) High-frequency oscilloscope (Tektronix Type 545) $\ddagger$ fitted with plug-in unit (Type 53/54 L).

Fig. 1 (opposite) shows the ultrasonic equipment $(a)$ and the high-frequency oscilloscope (b). The former is connected to the latter as described by Oksala and Lehtinen (1960). The echogram of the eye is seen on the ultrasonic screen macroscopically, the acoustic magnification being about $\times 4$. The oscilloscope screen simultaneously shows the echogram microscopically, magnified in the direction of both axis $x$ and axis $y$. If the magnification of the Tektronix time

* Received for publication May 25, 1960

$\dagger$ Manufactured by Dr. J. u. H. Krautkrämer, Gesellschaft für Elektrophysik, Cologne, Germany.

$\ddagger$ Manufactured by Tektronic Inc., Portland, Oregon, U.S.A. 
(b)

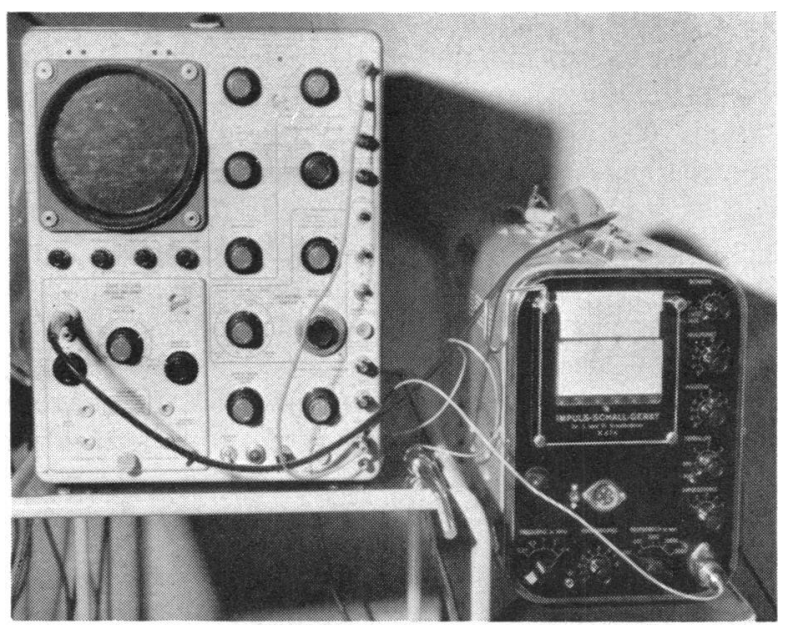

(a)

FIG. 1.-Ultrasonic apparatus (a) and high-frequency oscilloscope (b).

axis is 80 times that of the ultrasound time axis, the echogram can be continually magnified by as much as 320 . This means that the diameter of the eye can be lengthened out on the Tektronix scale to as much as $8 \mathrm{~m}$. In practice, however, magnifications from $10 \times$ to $50 \times$ are sufficient.

Crystals with the frequencies 6 and $18 \mathrm{Mhz}$ (Fig. 2) have been used in these investigations. Each has a diameter of $5 \mathrm{~mm}$., and they can be fitted with plexiglass frames, by means of which the sonic waves may be guided exactly axially, if so desired. Since the 6-Mhz crystal has a higher power of penetration, it is used for examining the intra-ocular and posterior parts of the eye, the 18-Mhz crystal being mainly used for examining the anterior and intra-ocular parts.

Fig. 2.-Crystals of $6 \mathrm{Mhz}$ (above) and $18 \mathrm{Mhz}$ (below). Both have a diameter of $5 \mathrm{~mm}$. They can be fitted with a plexiglass frame, which is shown both detached and fixed to the crystal.

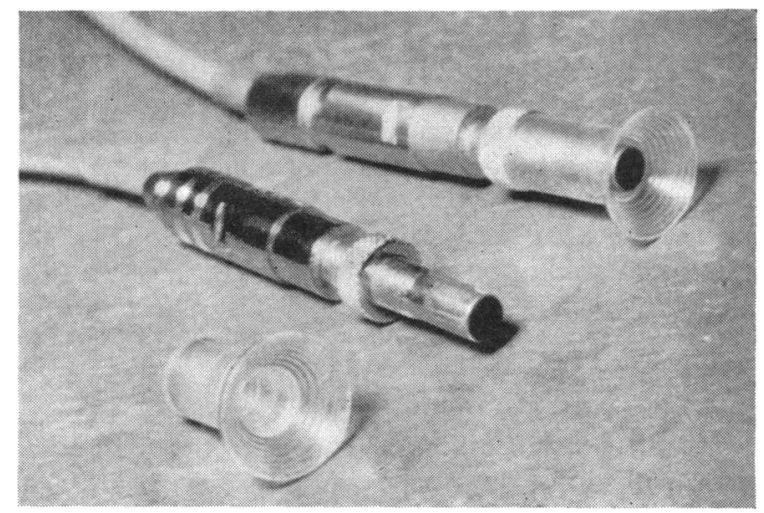

\section{Method of Examination}

The eye is anaesthetized with 3 per cent. Cornecain drops (Hoechst), after which a 1 per cent. methylcellulose solution is pipetted into the eye. The crystal is 
gently pressed against the surface of the eye, and echograms appear on the scales of both the ultrasonic equipment and the oscilloscope. By changing both the point of contact and the direction of the crystal, it is possible to examine all parts of the eye. Photographs are taken of the echograms visualized on the screen. So far the two screens have been photographed one after the other, and the echograms so obtained were not completely identical in form. The movements of the examiner's hand and of the eye cause changes in the echogram during the examination, even if one tries to keep the crystal perfectly still, but if the two screens were photographed simultaneously echograms would be obtained of the same shape but of a different magnitude. The differences which arise when the macroscopic and microscopic echograms are photographed one at a time have practical significance, since in any event a long series of echograms are necessary for diagnosis.

\section{Case Report}

A woman aged 42 had noticed that the visual acuity in the left eye had begun to deteriorate slowly for about 2 months. Since the eye was free from pain and not reddened, she had not sought medical aid immediately.

Examination.-On March 3, 1960, the visual acuity in the right eye was, 1.0 and in the left $\mathbf{0} \cdot 25$. The right eye was normal.

The left eye showed no injection, and the anterior parts of the eye and the vitreous were asymptomatic. In the fundus a large tumour was seen above temporally. It was about 2 disc diameter wide and the height measured with the ophthalmoscope was about $4 \mathrm{~mm}$. The surface of the tumour was compact and was pigmented in several places. The retina adhered to the surface of the tumour, and was oedematous between the tumour and the macula. A slight foveal tear could be distinguished.

Ultrasonic Examination.-Since, by means of the ophthalmoscope and the slit lamp, it was not possible to explore beyond the surface of the tumour, an examination was made with a frequency of $6 \mathrm{Mhz}$, the impulse power of the ultrasonic equipment being 5 , and amplification 10. Several echograms showed acoustically that subretinally there was heterogeneous tissue and not fluid. In numerous echograms the subretinal tumour tissue reflected a group of echoes of different height but chiefly low, which ended in a higher echo from the posterior wall of the globe.

Fig. 3 (opposite) shows two typical echograms reflected from the direction of the tumour and photographed on the screen of the ultrasonic equipment. On the left there is a high initial impulse followed by the zero-line typical of an undamaged vitreous, reaching as far as the echoes reflected by the tumour. In Fig. 3A the arrow marks the beginning of a group formed of only three low tumour echoes which ends in the higher echo seen on the right which is given off by the posterior wall of the globe. In Fig. 3B the tumour reflects only two echoes, which are higher and broader than those seen in Fig. 3A.

Fig. 4 (opposite) presents two typical echograms emitted by the tumour and photographed on the Tektronix screen. In Fig. 4A the acoustic magnification is $\times 10$ and in Fig. 4B it is $\times 20$. Fig. 4A shows on the right an echo from the posterior wall of the globe with three lower echo peaks in front of it. These three echoes prove that immediately in front of the posterior wall there is an acoustically heterogeneous tissue about $3 \mathrm{~mm}$. high. Fig. 4B shows one low but broad echo just in front of the echo from the posterior wall.

This eye was enucleated on March 15, 1960, and the histopathological examination demonstrated that the tumour was a melanoma of the choroid. 


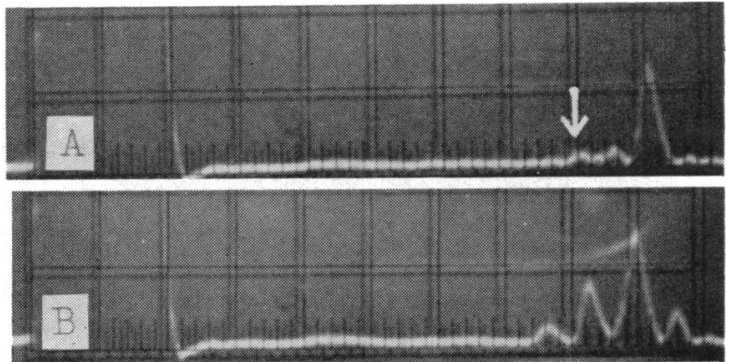

Fig. 3A and B.-Two typical echograms reflected from the direction of the tumour on screen of ultrasonic equipment. (Left) A high initial impulse followed by a zeroline typical of the undamaged vitreous, the echo from the posterior wall being seen on the right as a higher peak. (A) A group of low echoes (arrow) emitted by tumour and continuing as far as echo from posterior wall. (B) Tumour reflects only two echo peaks. Acoustic magnification $\times 4$.
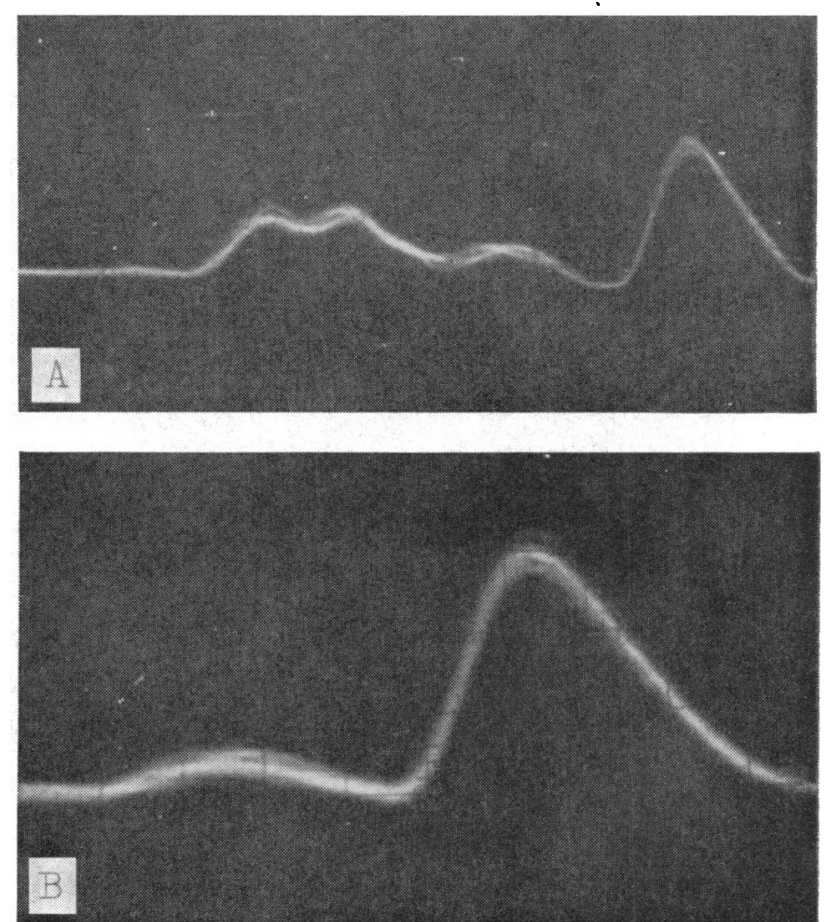

FIG. 4A and B.-Two echograms given off by tumour on screen of oscilloscope. Echo from posterior wall, on the right, with three echoes (A) and one echo (B) emitted by the tumour on the left. Acoustic magnification $\times 10(\mathrm{~A})$ and $\times 20(\mathrm{~B})$.

\section{Discussion}

In this case both the macroscopic and the microscopic ultrasound examinations clearly indicated that tissue was present subretinally, which had a 
rapidly and markedly varying acoustic resistance. Had there been only fluid, vitreous, or blood subretinally, the clear zero-line seen on the echogram after the echo given off by the retina would have continued as far as the echo from the posterior wall of the globe. We could not have obtained such a reliable idea of the subretinal space by any other technique of clinical examination. Further advantages of the ultrasonic method of examination are the absence of pain to the patient and of danger to the eye, and the ease with which it can be carried out.

Previously I have used only macroscopic ultrasonic examination in diagnosing intra-ocular tumours; the clinical diagnosis could be verified by this means in most cases, but microscopic ultrasonic examination (acoustic biomicroscopy) is an even more reliable method of examination by ultrasound. When tumour tissue contains oedema, necrosis, and haemorrhages, only very low echoes are reflected in several directions of investigation. In Coats's disease the echoes in numerous echograms were so low that they could hardly be visualized on the screen. The magnification of the echogram is very helpful particularly when the echoes are low, and it is also useful in showing two echoes separately which may appear as one macroscopically. Acoustic biomicroscopy has even provided crude data on the microscopical structure of the object under examination.

So far intra-ocular tumours have mainly been examined with a crystal of $6 \mathrm{Mhz}$, which gave good penetration of the tumour by ultrasonic waves. When the acoustic resistance and absorption of the tumour were lower, a frequency of $18 \mathrm{Mhz}$ was used and the resolving limit of acoustic biomicroscopy was about $200 \mu$.

\section{Summary}

Acoustic biomicroscopy has been used in the diagnosis of melanoma of the choroid. The equipment comprised an ultrasonic apparatus and a highfrequency oscilloscope which magnifies the echogram visualized on the screen. The echogram can thus be seen simultaneously both macroscopically and microscopically. The high magnification makes it possible to visualize low echo peaks more clearly and to distinguish those which lie very close together, thus improving the reliability of the ultrasound method of examination.

\section{REFERENCES}

Baum, G., and Greenwood, I. (1958). A.M.A. Arch. Ophthal., 60, 263.

(1960). Amer. J. Ophthal., 49, 249.

MundT, G. H., and HuGHES, W. F. (1956). Ibid., 41, 488.

OKSALA, A. (1958). Nord. Med., 59, 721.

- (1959a). Acta ophthal. (Kbh.), 37, 132.

(1959b). Brit. J. Ophthal., 43, 408.

and Lehtinen, A. (1957). Ophthalmologica (Basel), 134, 387. (1960). Acta ophthal. (Kbh.), 38, 19. 